\title{
Erratum: Three-Nucleon Low-Energy Constants from the Consistency of Interactions and Currents in Chiral Effective Field Theory [Phys. Rev. Lett. 103, 102502 (2009)]
}

\author{
Doron Gazit, Sofia Quaglioni, and Petr Navrátil
}

(Received 27 November 2018; published 15 January 2019)

DOI: 10.1103/PhysRevLett.122.029901

It was recently brought to our attention [1] that Eq. (2) contains an erroneous factor of -4 in the coefficient multiplying the $c_{D}$ low-energy constant (LEC). The correct expression for the strength $\hat{d}_{R}$ of the contact term of the meson-exchange currents (MEC) is given by

$$
\hat{d}_{R}=-\frac{M_{N}}{4 \Lambda_{\chi} g_{A}} c_{D}+\frac{1}{3} M_{N}\left(c_{3}+2 c_{4}\right)+\frac{1}{6} .
$$

This mistake affects the determination of the LEC $c_{D}$ entering the one-pion exchange plus the contact term of the threenucleon $(N N N)$ force at next-to-next-to-leading order $\left(\mathrm{N}^{2} \mathrm{LO}\right)$ from the triton $\beta$ decay, i.e., the results shown in Fig. 3. The correct results are shown in the figure below, where the shaded area represents a $2 \sigma$ tolerance band. Agreement with the empirical value based on a $2 \sigma$ error bar is found for $c_{D}=0.83 \pm 0.24$. The corresponding $c_{E}$ values are given by $c_{E}=-0.052_{-0.034}^{+0.033}$. As in the original Letter, the computed $\left\langle E_{1}^{A}\right\rangle_{\text {theor }} /\left\langle E_{1}^{A}\right\rangle_{\text {emp }}$ ratio is rather insensitive to the $N N N$ force. However, due to the roughly factor of -4 rescaling of $c_{D}$, the difference with respect to results obtained using $A=3$ wave functions produced by the phenomenological AV18 nucleon-nucleon $(N N)$ potential and by the next-to-next-to-next-toleading order $\left(\mathrm{N}^{3} \mathrm{LO}\right) N N$ potentials of Epelbaum et al. [30] is now amplified. Similarly, calculations (without $N N N$ force) carried out by setting $c_{4}$ to $3.4 \mathrm{GeV}^{-1}$ in the axial-vector current, while the $A=3$ wave functions are obtained from the $\mathrm{N}^{3} \mathrm{LO} N N$ potential of Ref. [22] produces a shift of about -1.2 in the $c_{D}$ values.

Table I displayed the ${ }^{3} \mathrm{H},{ }^{3} \mathrm{He}$, and ${ }^{4} \mathrm{He}$ ground state (g.s.) energies and point-proton radii obtained using the $\mathrm{N}^{3} \mathrm{LO} N N$ potential [22] with and without the local $\mathrm{N}^{2} \mathrm{LO} N N N$ interaction [23] with $c_{D}=-0.2$ and $c_{E}=-0.205$. The updated table

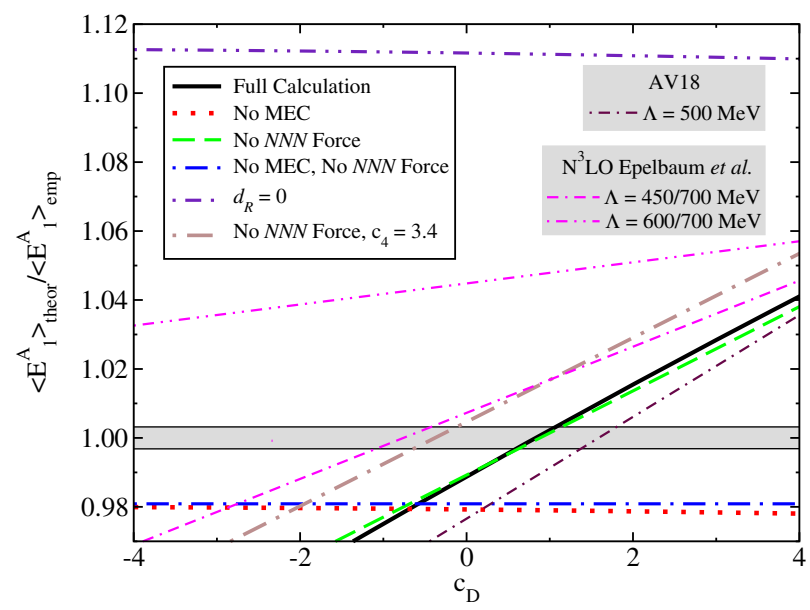

FIG. 3. The ratio $\left\langle E_{1}^{A}\right\rangle_{\text {theor }} /\left\langle E_{1}^{A}\right\rangle_{\text {emp }}$ using the $\mathrm{N}^{3} \mathrm{LO} N N$ potential [22] with and without the local $\mathrm{N}^{2} \mathrm{LO} N N N$ interaction [23], and the axial current with and without MEC $\left(c_{D}\right.$ and $c_{E}$ are varied along the averaged trajectory of Fig. 2). The shaded area represents a $2 \sigma$ tolerance band. Also shown are the results for the phenomenological AV18 potential (with $\Lambda=500 \mathrm{MeV}$ imposed in the current), and for the $\mathrm{N}^{3} \mathrm{LO} N N$ potential of Epelbaum et al. [30] (with $\Lambda=450,600 \mathrm{MeV}$, and a $700 \mathrm{MeV}$ spectral-function cutoff in the two-pion exchange). 
TABLE I. Calculated ${ }^{3} \mathrm{H},{ }^{3} \mathrm{He}$, and ${ }^{4} \mathrm{He}$ g.s. energies (in $\mathrm{MeV}$ ) and point-proton radii (in fm), obtained using the $\mathrm{N}^{3} \mathrm{LO} N N$ potential [22] with and without the local $\mathrm{N}^{2} \mathrm{LO} N N N$ interaction [23] with $c_{D}=0.83$ and $c_{E}=-0.052$, compared to experiment.

\begin{tabular}{|c|c|c|c|c|c|c|}
\hline & \multicolumn{2}{|c|}{${ }^{3} \mathrm{H}$} & \multicolumn{2}{|c|}{${ }^{3} \mathrm{He}$} & \multicolumn{2}{|c|}{${ }^{4} \mathrm{He}$} \\
\hline & $E_{\text {g.s. }}$ & $\left\langle r_{p}^{2}\right\rangle^{1 / 2}$ & $E_{\text {g.s. }}$ & $\left\langle r_{p}^{2}\right\rangle^{1 / 2}$ & $E_{\text {g.s. }}$. & $\left\langle r_{p}^{2}\right\rangle^{1 / 2}$ \\
\hline$N N$ & $-7.852(2)$ & $1.651(5)$ & $-7.124(2)$ & $1.847(5)$ & $-25.39(1)$ & $1.515(2)$ \\
\hline$N N+N N N$ & $-8.473(2)$ & $1.607(5)$ & $-7.729(2)$ & $1.787(5)$ & $-28.38(2)$ & $1.469(5)$ \\
\hline Expt. & -8.482 & 1.60 & -7.718 & 1.77 & -28.296 & $1.467(13)[31]$ \\
\hline
\end{tabular}

for the new set of LECs, $c_{D}=0.83$ and $c_{E}=-0.052$ is shown above. The ${ }^{4} \mathrm{He}$ g.s. energy and point-proton radius for the lower $(0.59,-0.086)$ and upper $(1.07,-0.018)$ bounds of $c_{D}$ and $c_{E}$ are, respectively, $E_{\text {g.s. }}=-28.41(2)$, $\left\langle r_{p}^{2}\right\rangle^{1 / 2}=1.467(5)$, and $E_{\mathrm{g} . s .}=-28.35(2),\left\langle r_{p}^{2}\right\rangle^{1 / 2}=1.471(5)$. The other results and conclusions of the original Letter remain unaffected.

We thank E. Epelbaum, S. Pastore, R. Schiavilla, and K. Wendt for useful discussions. Computing support for this work came from the LLNL institutional Computing Grand Challenge program. Prepared in part by LLNL under Contract DEAC52-07NA27344. This material is based upon work supported by the U.S. Department of Energy, Office of Science, Office of Nuclear Physics, under Work Proposal No. SCW1579, the LDRD project 18-ERD-008, and by the NSERC Grants No. 401945-2011 and No. SAPIN-2016-00033. TRIUMF receives federal funding via a contribution agreement with the National Research Council of Canada.

[1] R. Schiavilla (private communication). 